\title{
Creatinine Reduction Ratio is a Prognostic Factor for Acute Kidney Injury Following Cardiac Surgery with Cardio Pulmonary Bypass: A Single Center Retrospective Cohort Study.
}

akiko anzai

Yokohama City University Hospital

shunsuke takaki ( $\sim$ shunty5323@gmail.com )

Yokohama City University https://orcid.org/0000-0001-5675-6657

Nobuyuki Yokoyama

Yokohama City University

Shizuka Kashiwagi

Yokohama City University: Yokohama Shiritsu Daigaku

Masashi Yokose

Yokohama City University: Yokohama Shiritsu Daigaku

Takahisa Goto

Yokohama City University: Yokohama Shiritsu Daigaku

\section{Research Article}

Keywords: Reduction of creatinine, Acute kidney injury, Cardiopulmonary bypass

Posted Date: July 30th, 2021

DOI: https://doi.org/10.21203/rs.3.rs-716926/v1

License: (c) (i) This work is licensed under a Creative Commons Attribution 4.0 International License.

Read Full License 


\section{Abstract}

Background: Acute kidney injury (AKI) after cardiac surgery is common complication, and it is known as risk of death. In the previous study, we reported creatinine reduction ratio (CRR) can be useful as a prognostic factor of AKI. Primary outcome of this study was determination of predictor of AKI after surgery with using perioperative information. Secondary outcome was the reliability of CRR for short and long term outcome among the different nation.

Methods: We retrospectively collected cardiac surgical patients with cardiopulmonary bypass from electrical health record. Patients were excluded with insufficient data. AKI was defined by the AKIN and RIFLE criteria, (1) increment of creatinine $\geqq 0.3 \mathrm{mg} / \mathrm{dl}$ (2); increment of creatinine $\geqq 150 \%$. Patients were divided by AKI and non-AKI group. We analyzed two group about the preoperative patients' data and operative information. CRR was calculated as follows: (preoperative creatinine-postoperative creatinine)/preoperative creatinine.

Results: 225 patients data were collected from electrical health record, and analysed. The prognostic factor of AKI-CS was surgery time, CPB time, aorta clamp time, platelet transfusion, and CRR $<20 \%$. In the multivariable logistic analysis, CRR was an independent predictor of AKI. (adjusted odds ratio 0.90[0.870.93] $p<0.001$ ) However, there were not significant difference in CRR about the rate of new onset of chronic kidney disease (CKD).

Conclusions: After cardiac surgery with cardiopulmonary bypass, the CRR has a good diagnostic power for predicting perioperative AKI. However, we cannot use it as a prognostic factor for long term period.

\section{Background}

Acute kidney injury(AKI) after cardiac surgery (AKI-CS) is common complication with high incidence rate up to $30 \%$ (1), and one of the strong risk factors for increasing mortality .(2) Andrea and colleague reported even minimal changes of serum creatinine within 48 hours after cardiac surgery increased mortality.(3) However, in cardiac surgery with cardiopulmonary bypass (CPB), serum creatinine immediately after surgery was normally decreased due to fluid balance. Therefore, it takes time until serum creatinine is maximally developed after initiation of diuretic phase. Failure to recognize AKI because of fluid overload may lead to a relative increase in mortality in those with fluid overload who (by conventional creatinine criteria) do not have AKI or have mild AKI, since minimal increases in creatinine are associated with significant increases in mortality. $(3,4)$ Renal injury itself further increases the risk of developing severe non-renal complications that may lead to death. $(5,6)$

Our hypothesis is that minimal change of serum creatinine post cardiac surgery with CPB might affect postoperative renal function. In our previous study, we calculated CRR with pre and post creatinine value as following calculation. (7)

$\mathrm{CRR}=\{($ Post sCr - Pre sCr $) / \mathrm{Pre} s \mathrm{Cr}\} * 100$ 
Post sCr : post creatinine value, Pre sCr : pre creatinine value

CRR might be useful predictor for AKI-CS detection, however in our previous study, background of patients and anesthetic information were not included to analysis. Furthermore, previous study did not elucidate whether CRR can predict long term outcome of renal function in addition to postoperative AKI.

In this study, therefore, we reevaluated reliability of CRR for short and long term outcome with using more perioperative information. Primary outcome of this study was determination of predictor of perioperative AKI-CS after surgery with using perioperative information. Our secondary outcome was whether perioperative serum creatinine $(\mathrm{sCr})$ transition and $\mathrm{AKI}-\mathrm{CA}$ affect long term outcome of renal function.

AKI is defined as increasing of creatinine value within 24 to 48 hours after operation according to AKIN and RIFLE criteria. Early prediction of AKI is defined as RIFLE criteria at post operative day 1 or 2.

\section{Methods}

\section{Study Population}

This present study was approved by institution review board at Yokohama City University Hospital, and registered at University Medical Information Network (UMIN ID: 000011927). As this study is retrospective study, patient consent was not obtained. We retrospectively collected data of patients who underwent cardiac surgery, such as coronary artery bypass grafting and valve replacement, with cardiopulmonary bypass at Yokohama city university hospital from 2009 to 2014 . We collected medical information of 299 patients, 74 patients were excluded with insufficient data. Therefore, 225 patients were included to present study.

\section{Factor Measurement}

In the preoperative patient data, age, hypertension, diabetes mellitus, renal function, heart disease, stroke, and respiratory function were collected. Anesthetic time, operation time, CPB time, aortic clamp time, transfusion, and perioperative creatinine value were also collected as operative information. Serum creatinine was measured immediately after surgery in the operation room or ICU, and compared that of preoperative.

AKI was defined by the AKIN and RIFLE criteria using the elevation of sCr from baseline within 48-hour after surgery as following two methods: (1) increment of creatinine $\geqq 0.3 \mathrm{mg} / \mathrm{dl}$ (2); increment of creatinine $\geqq 150 \%$. (8)

In our previous study, CRR was detected as predictor of CS-AKI in cardiac surgery under CPB. CRR was defined perioperative change ratio of serum creatinine pre and post CPB. In the CPB, blood dilution is occured due to CPB priming volume. Therefore, fluctuation of serum creatinine is happened during perioperative state. Perioperative CRR with pre and post creatinine value was also calculated as following calculation. 
Patients were divided to two groups as AKI-CS group and non-AKI-CS group according to AKIN and RIFLE criteria. Patients with insufficient data were excluded from this study. Predictor of AKI after cardiac surgery was determined with using perioperative information. In the previous studies about AKI-CS, there are some risk factors (ex. Age, woman, cardiac failure, history of cardiac surgery, chronic obstructive pulmonary disease, peripheral arterial disease, diabetes mellitus, renal function injury, emergency, the use of intra aortic balloon pumping). (2-4, 9-13) These confounding variables as AKI-CS risk factor were included to multivariable analysis in addition to CRR $<20 \%$.

Furthermore, we analyzed long term kidney function. Chronic renal function was also evaluated with using eGFR calculated by modification of diet in renal disease study (MDRD) at 3 to 6 month after operation. CRR and perioperative AKI-CS incidence were evaluated as reliable predictor of chronic kidney disease.

\section{Statistics}

Quantitative analysis of background and perioperative information were described as median with interquartile range. The univariable analysis of patient's information was performed between AKI-CS and Non-AKI-CS group as following method. Categorical data was analyzed by Fisher's exact test, and continuous data was analyzed by Mann-Whitney $U$ test. The area under the receiver operating characteristic curve was calculated for CRR $<20 \%$ in the diagnosis of AKI. Various risk factors influence incidence of AKI-CS. (9) The multivariable logistic analysis for predictor of AKI incidence with using predictors. Predictors were selected according to risk factors which were reported by previous research. Akaike's information criterion (AIC) stepwise procedure was used to select final confounding variables for multivariable logistic analysis with lowest value of the AIC.

For two-tailed tests, a P-value of $<0.05$ was considered statistically significant. Statistical analyses were performed using GraphPad Prism 6 for Mac OS X version 6.9b (GraphPad Software, Inc., San Diego, CA, USA) and R 2.13.0 statistical software (R Foundation for Statistical Computing, Vienna, Austria).

\section{Results}

We collected medical information of 299 patients, 74 patients were excluded with insufficient data (e.g. preoperative creatinine value, operation data). Therefore, 225 patients were included to present study. The patients were divided two groups, 97 patients (57\%) were AKI-CS group and 128 patients (43\%) were nonAKI-CS group. Area under the receiver operating characteristic curve for AKI-CS prediction was 0.714 $(0.650-0.778, p<0.001)$ CRR $15 \%$ and $20 \%$ of CRR were optimal threshold value with good diagnostic power. (Figure.1)(7)

\section{Univariate analysis}


Surgery time, CPB time, aorta clamp time, platelet transfusion, and CRR $<20 \%$ and $15 \%$ were significant difference between AKI and non-AKI group. (Table.1)

Table.1: Background and perioperative information in total, AKI, and non-AKI group.

\begin{tabular}{|c|c|c|c|c|}
\hline & $\begin{array}{c}\text { Total } \\
n=225\end{array}$ & $\begin{array}{c}\text { AKI } \\
n=97\end{array}$ & $\begin{array}{c}\text { Non-AKI } \\
n=128\end{array}$ & $P$ value \\
\hline$(y r)$ & $66(49-75)$ & $67(50-74)$ & $64.5(48-75.25)$ & 0.69 \\
\hline (male, \%) & $148(65.8 \%)$ & 65 (67.0\%) & $83(64.8 \%)$ & 0.78 \\
\hline Height & $1.64(1.56-1.7)$ & $1.63(1.55-1.68)$ & $17(11.6 \%)$ & 0.57 \\
\hline Weight & $59.2(49.5-66.5)$ & $58.8(49.23-66.20)$ & $56(38.3 \%)$ & 0.90 \\
\hline Body Mass Index (BMI) & $22.1(19.4-24.3)$ & $22.32(19.59-24.60)$ & $30(20.5 \%)$ & 0.54 \\
\hline ASA risk & $3(2-3)$ & $3(2-3)$ & $3(2-3)$ & 0.86 \\
\hline Urgent $\quad(n, \%)$ & $29(12.8 \%)$ & $8(7.5 \%)$ & $20(13.7 \%)$ & 0.16 \\
\hline HT $\quad(n, \%)$ & $89(39.5 \%)$ & 37 (38.1\%) & $52(40.6 \%)$ & 0.78 \\
\hline$D M \quad(n, \%)$ & $34(15.1 \%)$ & $18(18.5 \%)$ & $16(12.5 \%)$ & 0.26 \\
\hline COPD $(n, \%)$ & $1(0.04 \%)$ & $1(1.03 \%)$ & $0(0 \%)$ & 0.43 \\
\hline Preope Diuretics $(n, \%)$ & $61(27.1 \%)$ & $28(28.8 \%)$ & $33(25.7 \%)$ & 0.65 \\
\hline ARB usage $\quad(n, \%)$ & $70(31.1 \%)$ & $33(34.0 \%)$ & $37(28.9 \%)$ & 0.51 \\
\hline \multicolumn{5}{|l|}{ Operation } \\
\hline CABG & 32 (27.1\%) & $15(14.2 \%)$ & $17(11.6 \%)$ & 0.57 \\
\hline Valve $\quad(n, \%)$ & $95(42.2 \%)$ & $39(36.8 \%)$ & $56(38.3 \%)$ & 0.90 \\
\hline GreatArtery $\quad(n, \%)$ & $56(24.8 \%)$ & 26 (24.5\%) & $30(20.5 \%)$ & 0.54 \\
\hline Others (Congenital) $(n, \%)$ & 41 (18.2\%) & $18(16.5 \%)$ & $23(15.8 \%)$ & 0.86 \\
\hline Combined $\quad(n, \%)$ & $28(12.4 \%)$ & $8(7.5 \%)$ & $20(13.7 \%)$ & 0.16 \\
\hline Surgery Time (hour) & 7.63(5.96-9.83) & $\underline{8.21(6.20-10.25)}$ & $\underline{7.38(5.63-9.41)}$ & $\underline{0.003}$ \\
\hline CPB Time (hour) & $\underline{3.61(2.71-4.68)}$ & $3.9(3.05-4.91)$ & $3.31(2.53-4.4)$ & $\underline{0.03}$ \\
\hline Ao Clamp (hour) & $\underline{2.38(1.55-3.13)}$ & $\underline{2.61(1.81-3.43)}$ & $\underline{2.25(1.45-2.86)}$ & $\underline{0.03}$ \\
\hline Anesth Tme (hour) & $9.46(7.73-11.75)$ & $\underline{10.2(8.21-12.1)}$ & $9.15(7.46-11.3)$ & $\underline{0.004}$ \\
\hline In Out Balance (L) & $2.70(0.899-5.18)$ & $2.48(0.64-4.70)$ & $2.95(1.18-5.54)$ & 0.51 \\
\hline $\mathrm{RBC}$ & $1120(560-1960)$ & $1120(560-1680)$ & $1120(560-1960)$ & 0.37 \\
\hline$(\mathrm{ml})$ & $\underline{500(250-750)}$ & $\underline{500(250-750)}$ & $\underline{250(500-500)}$ & $\underline{0.02}$ \\
\hline (ml) & $1200(720-2160)$ & $1440(720-2160)$ & $960(720-2010)$ & 0.58 \\
\hline Crystalloid & $3.75(2.4-12.38)$ & $3.3(2.3-7.33)$ & $4.5(2.4-13.2)$ & 0.08 \\
\hline Colloid & $550(500-1000)$ & $500(500-1000)$ & $650(500-1000)$ & 0.92 \\
\hline Bleeding & $1.89(1.06-3.90)$ & $1.84(0.791-3.97)$ & $1.91(1.1-3.65)$ & 0.57 \\
\hline Urine & $2.56(1.66-3.45)$ & $2270(1657-3450)$ & $2655(1665-3432)$ & 0.31 \\
\hline Diuretic usage $\quad(n, \%)$ & $38(16.8 \%)$ & $21(34.9 \%)$ & $17(13.3 \%)$ & 0.08 \\
\hline CRR $<20 \% \quad(n, \%)$ & $\underline{170(75.6 \%)}$ & $\underline{85(87.6 \%)}$ & $\underline{85(66.4 \%)}$ & $\leq 0.001$ \\
\hline$C R R<15 \% \quad(n, \%)$ & $148(83.5 \%)$ & $80(82.4 \%)$ & $\underline{68(53.1 \%)}$ & $\leq 0.001$ \\
\hline
\end{tabular}

ASA: American Society of Anesthesiologists, HT: Hyper tension, DM: Diabetes Mellitus, COPD: Chronic Obstructive Pulmonary Disease, ARB: Angiotensin II Receptor Blocker, CABG: Coronary Artery Bypass 
Graft, CPB: Cardio Pulmonary Bypass, Ao: Aorta, RBC: Red Blood Cell, PC: Platelet Concentrate, FFP: Flesh Frozen Plasma, CRR: Creatinine Reduction Ratio.

There were no significant difference about Apache II score, ICU duration, hospital stay between two groups. However, CKD newly development in the patients without previous chronic kidney disease was significant difference between two groups. (Table.2)

Table.2: Postoperative information between AKI and non AKI after cardiac surgery with cardiopulmonary bypass.

\begin{tabular}{|llll|}
\hline & AKI-CS group & Non-AKI-CS group & p-value \\
& $\mathbf{n}=\mathbf{1 0 6}$ & $\mathbf{n}=146$ & \\
\hline APACHE II & $17.6 \pm 8.0$ & $18.1 \pm 7.7$ & 0.58 \\
\hline Ventilator Free Days (day) & $4.8 \pm 4.2$ & $4.2 \pm 2.9$ & 0.17 \\
\hline ICU duration (day) & $4.8 \pm 4.2$ & $4.2 \pm 2.9$ & 0.17 \\
\hline Hospital Stay (day) & $42.0 \pm 90.5$ & $31.2 \pm 22.1$ & 0.17 \\
\hline & AKI-CS group & Non-AKI-CS group & \\
& $3-6$ month & $3-6$ month & \\
\hline CKD development $(n, \%)$ & 18 (18/51, 35.3\%) & $5(5 / 50,10 \%)$ & 0.004 \\
\hline
\end{tabular}

APACHE II (Acute physiology and chronic health evaluation) II, CKD: Chronic kidney disease

CKD development: 101 patients with preoperative normal renal function were assessed for newly chronic kidney development in postoperative 3-6 months.

\section{Multivariable analysis}

In the multivariable logistic analysis, CRR $<15 \%$ was associated with the incidence of perioperative AKICS compared CRR < 20\%.(adjusted odds ratio 0.90[0.87-0.93] $p<0.001$ ) (Table. 3)

Table.3: Multivariable logistic regression analysis of prognostic factors for perioperative AKI incidence after cardiac surgery.

\begin{tabular}{lccc}
\hline & Adjusted Odds Ratio & 95\% Confidence Interval & P value \\
\hline APACH II & 0.95 & $0.90-1.00$ & 0.07 \\
CPB time & 2.32 & $1.45-3.73$ & $<0.001$ \\
CRR & 0.90 & $0.87-0.93$ & $<0.001$ \\
Preope eGFR & 1.02 & $1.00-1.03$ & 0.02 \\
Surgery time & 1.29 & $1.04-1.61$ & 0.02 \\
\hline
\end{tabular}


APACHE II (Acute physiology and chronic health evaluation) II, CPB: Cardiopulmonary bypass, CRR: Creatinine Reduction Ratio, eGFR: estimated glomerular filtration ratio.

CRR (CRR $<20 \%$ and $<15 \%$ ) might be good prognostic factor with sensitivity $87.6 \%$ and $82.5 \%$, specificity $33.6 \%$ and $46.9 \%$, positive predictive value 0.50 and 0.54 , negative predictive value 0.78 and 0.78 , respectively. (Table. 4)

Table.4: Diagnostic power of creatinine reduction ratio of $20 \%$ and $15 \%$ for AKI incidence and diagnostic power of CRR $<20 \%$, CRR $<15 \%$ and perioperative AKI for new CKD development.

\begin{tabular}{|c|c|c|c|c|c|}
\hline Predictor & CRR $<20 \%$ & CRR $<15 \%$ & CRR $<20 \%$ & CRR $<15 \%$ & Perioperative AKI \\
\hline \multirow[t]{3}{*}{ Outcome } & AKI incidence & AKI incidence & $\begin{array}{c}\text { New CKD } \\
\text { development }\end{array}$ & $\begin{array}{c}\text { New CKD } \\
\text { development }\end{array}$ & $\begin{array}{c}\text { New CKD } \\
\text { development }\end{array}$ \\
\hline & $\begin{array}{l}\text { AKI vs. non-AKI } \\
\qquad(n=225)\end{array}$ & $\begin{array}{l}\text { AKI vs. non-AKI } \\
\qquad(n=225)\end{array}$ & $\begin{array}{l}\text { CKD vs. non-CKD } \\
\qquad(n=101)\end{array}$ & $\begin{array}{c}\text { CKD vs. non-CKD } \\
\quad(n=101)\end{array}$ & $\begin{array}{c}\text { CKD vs. non-CKD } \\
\qquad(n=101)\end{array}$ \\
\hline & 97 vs. 128 & 97 vs. 128 & 23 vs. 78 & 23 vs. 78 & 23 vs. 78 \\
\hline $\begin{array}{l}\text { Unadjusted Odds } \\
\text { ratio }\end{array}$ & $8.08(2.75-23.7)$ & $4.15(2.22-7.78)$ & $0.36(0.07-1.74)$ & $0.86(0.25-2.99)$ & $4.91(1.65-14.57)$ \\
\hline Sensitivity & $87.6 \%(79.4-93.4)$ & $82.5 \%(73.4-89.5)$ & $86.7 \%(66.4-97.2)$ & $82.6 \%(61.2-95.1)$ & $78.3 \%(56.3-92.5)$ \\
\hline Specificity & $33.6 \%(25.5-42.5)$ & $46.9 \%(38.0-55.9)$ & $5.1 \%(1.4-12.6)$ & $15.4 \%(8.2-25.3)$ & $57.7 \%(46.0-68.8)$ \\
\hline PPV & $0.50(0.42-0.57)$ & $0.54(0.46-0.62)$ & $0.21(0.13-0.31)$ & $0.22(0.14-0.33)$ & $0.35(0.22-0.50)$ \\
\hline NPV & $0.78(0.65-0.88)$ & $0.78(0.67-0.87)$ & $0.57(0.18-0.90)$ & $0.75(0.48-0.93)$ & $0.90(0.78-0.97)$ \\
\hline Relative risk & $4.58(1.79-11.7)$ & $2.45(1.57-3.82)$ & $0.49(0.19-1.27)$ & $0.89(0.35-2.28)$ & $3.53(1.42-8.78)$ \\
\hline$P$ value & $\leq 0.001$ & $\leq 0.001$ & 0.19 & 0.76 & $\underline{0.004}$ \\
\hline
\end{tabular}

CRR: Creatinine Reduction Ratio, CKD: Chronic Kidney Disease, AKI: Acute Kidney Injury, PPV: Positive Predictive Value, NPV: Negative Predictive Value

\section{Long-term results}

101 patients with preoperative normal renal function were assessed for newly CKD development in postoperative 3-6 months. 124 patients were excluded from evaluation due to insufficient data and preoperative CKD. In the patients with normal renal function before surgery, the rate of development of CKD was higher in the AKI-CS group compared to the non-AKI-CS group. (35.3\% [18/51] vs 10\% [5/50], $p=$ 0.004) (Table. 4)

\section{Discussion}

It is known that renal function injury affects the prognosis after cardiac surgery.(2) Several studies reported the risk factors of $\mathrm{AKI}$, for example, female gender, reduced left ventricular function, the presence of congestive heart failure, diabetes, chronic obstructive pulmonary disease, an elevated preoperative 
serum creatinine and so on.(14) Even minimum increase of serum creatinine value $(<0.3 \mathrm{~m} / \mathrm{dl}) \mathrm{may}$ increase the mortality rate.(3) Blood flow reduction may lead to cellular injury due to imbalance between oxygen delivery and demand(15), but the reason is not clear why the decrease of sCr protect AKI. Our previous study reported that CRR may be associated with AKI-CS. In this study, we reconsidered the perioperative information included CRR and also evaluated CRR as the predictors of long-term renal injury. In our data, Multivariable logistic analysis showed lower CRR is associated with perioperative AKI. CRR is useful to prevent AKI which may lead to CKD, so it is important to consider the treatment in early period by using CRR. To avoid the development of AKI, low-dose ANP and fenoldopam are recommended by low-quality evidence. Hydroxyethyl starches and hyperchloremic solution affect kidney function, so we should choice other solutions. (16)

It is unclear whether perioperative creatinine change may lead to CKD. Brown and colleague studied the relation between the term of $\mathrm{AKI}$ and the prognosis after cardiac surgery, and reported the mortality rate of postoperative 5 years is 1.66 times in patients with AKI for even 1-2 days. (16) AKI patients who recovered normal renal function before discharge have higher mortality than non-AKI patients. (17) In our study, there were not significant difference in CRR about the rate of new onset of CKD. Since the patients without data of postoperative 3-6 months were excludes, this may have something to the results. However, in the patients with normal renal function before surgery, the rate of development of CKD was higher in the AKI-CS group compared to the non-AKI-CS group. More than $90 \%$ of patients without AKI didn't progress to CKD for a long period after surgery, and AKI is one of the risk factors for CKD development. CRR is effective for predicting AKI in early period, so it is important to consider the treatment in early period by using CRR before next laboratory data is available, and initiation of renal protection strategy might prevent developed CKD in the long term after operation.

We have several limitations in this research. The first, urine output was not included in this present study. Kama and colleague reported, RIFLE without the urine output criteria significantly underestimated risk of $\mathrm{AKI}$ and delayed diagnosis of AKI in critical ill patients. (18) In the post cardiac surgery, urine output was strongly affected by administration of diuretics during anesthesia and cardiopulmonary bypass. Therefore, we excluded urine output from definition of AKI. Secondly, we calculated renal function by using MDRD which patient's age and serum biochemistry (Cre, BUN, Alb) effect. These factors are variable after cardiac surgery. Thirdly, this present study was conducted single center retrospective cohort study and we collected different type of surgery (only valve, CABG, congenital cardiac disease and aortic surgery). Furthermore, Chew and colleague reported that the risk of AKI incidence was differed according to race (19) and this may be the reason why the rate of AKI in our study was higher than in other studies. These results also may not be adapted to other medical center.

There are two good points of using CRR. First, we can predict AKI by blood test immediately after surgery. In the conventional method, it needs long time to define AKI because AKI is defined as increasing of $s C r$ value within 24 to 48 hours after operation. However, we can predict AKI by using CRR at the timing of termination of operation and consider the treatment in early period. Secondly, CRR can be calculated by 
only perioperative creatinine value and we can get it very easily and at a low cost. Most patients are checked by blood test after surgery, so additional test is not required.

\section{Conclusion}

CRR may be a useful predictor of perioperative AKI after cardiac surgery with a cardiopulmonary bypass , but may not be useful for the long-term outcome of renal function. Further studies are required to verify our findings. Perioperative AKI patients progress to CKD with high frequency for a long period.

\section{Abbreviations}

AKI

Acute kidney injury

CRR

creatinine reduction ratio

CKD

chronic kidney disease

AKI-CS

Acute kidney injury(AKI) after cardiac surgery

CPB

cardiopulmonary bypass

AIC

Akaike's information criterion

ASA

American Society of Anesthesiologists,

HT

Hyper tension

DM

Diabetes Mellitus

COPD

Chronic Obstructive Pulmonary Disease,

ARB

Angiotensin II Receptor Blocker

CABG

Coronary Artery Bypass Graft

CPB

Cardio Pulmonary Bypass

Ao

Aorta

RBC 
Red Blood Cell

PC

Platelet Concentrate

\section{Declarations}

Ethics approval and consent to participate

Yokohama City University committee of ethics approved this present study.

Consent for publication: not applicable

Availability of data and materials. The datasets during and/or analysed during the current study available from the corresponding author on reasonable request.

Funding: non

Competing interests. The authors declare that they have no competing interests.

\section{Authors' contributions:}

TS and AA contributed study design, analysis, wrote the manuscript, AA contributed data collection, cleaned data, and wrote the manuscript. YN, KS, YM and GT contributed overall direction and planning of this study. All authors read and approved the final manuscript."

\section{Acknowledgements.}

We thank all the research staff and the healthcare provider who supported our research.

\section{References}

1. Lagny M-G, Jouret F, Koch J-N, Blaffart F, Donneau A-F, Albert A, Roediger L, Krzesinski J-M, Defraigne $\mathrm{J}-\mathrm{O}$. Incidence and outcomes of acute kidney injury after cardiac surgery using either criteria of the RIFLE classification. BMC Nephrol. 2015;16:76.

2. Chertow GM, Levy EM, Hammermeister KE, Grover F, Daley J. Independent association between acute renal failure and mortality following cardiac surgery. Am J Med. 1998;104:343-8.

3. Lassnigg A, Schmidlin D, Mouhieddine M, Bachmann LM, Druml W, Bauer P, Hiesmayr M. Minimal changes of serum creatinine predict prognosis in patients after cardiothoracic surgery: a prospective cohort study. J Am Soc Nephrol. 2004;15:1597-605.

4. Takaki S, Shehabi Y, Pickering JW, Endre Z, Miyashita T, Goto T. Perioperative change in creatinine following cardiac surgery with cardiopulmonary bypass is useful in predicting acute kidney injury: a single-centre retrospective cohort study. Interact Cardiovasc Thorac Surg. 2015;21:465-9. 
5. Anderson RJ, O'brien M, MaWhinney S, VillaNueva CB, Moritz TE, Sethi GK, Henderson WG, Hammermeister KE, Grover FL, Shroyer AL. Renal failure predisposes patients to adverse outcome after coronary artery bypass surgery. VA Cooperative Study \#5. Kidney Int. 1999;55:1057-62. doi:10.1046/j.1523-1755.1999.0550031057.x.

6. Ryckwaert F, Boccara G, Frappier J-M, Colson PH. Incidence, risk factors, and prognosis of a moderate increase in plasma creatinine early after cardiac surgery. Crit Care Med. 2002;30:1495-8.

7. Thakar CV, Arrigain S, Worley S, Yared J-P, Paganini EP. A clinical score to predict acute renal failure after cardiac surgery. J Am Soc Nephrol. 2005;16:162-8.

8. von Wichert P, Morr H, Müller-Scheven D. Results in the treatment of allergic asthma using disodium cromoglycate. Med Welt. 1974 Feb 15;25(7):278-80.

9. Parolari A, Pesce LL, Pacini D, Mazzanti V, Salis S, Sciacovelli C, Rossi F, Alamanni F, Monzino Research Group on Cardiac Surgery Outcomes. Risk factors for perioperative acute kidney injury after adult cardiac surgery: role of perioperative management. Ann Thorac Surg. 2012;93:584-91.

10. Brown JR, Cochran RP, Leavitt BJ, Dacey LJ, Ross CS, MacKenzie TA, Kunzelman KS, Kramer RS, Hernandez F, Helm RE, Westbrook BM, Dunton RF, Malenka DJ, O'Connor GT, Northern New England Cardiovascular Disease Study Group. Multivariable prediction of renal insufficiency developing after cardiac surgery. Circulation. 2007;116(11 Suppl):1139-43.

doi:10.1161/CIRCULATIONAHA.106.677070.

11. Palomba H, de Castro I, Neto ALC, Lage S, Yu L. Acute kidney injury prediction following elective cardiac surgery: AneyKICS Score. Kid Int. 2007;72:624-31.

12. Wijeysundera DN, Karkouti K, Dupuis J-Y, Rao V, Chan CT, Granton JT, Beattie WS. Derivation and validation of a simplified predictive index for renal replacement therapy after cardiac surgery. JAMA. 2007;297:1801-9.

13. Aronson S, Fontes ML, Miao Y, Mangano DT, Investigators of the Multicenter Study of Perioperative Ischemia Research Group, Ischemia Research and Education Foundation. Risk index for perioperative renal dysfunction/failure: critical dependence on pulse pressure hypertension. Circulation. 2007;115:733-42. doi:10.1161/CIRCULATIONAHA.106.623538.

14. Mitchell H, Rosner MD, Okusa. Acute Kidney Injury Associated with Cardiac Surgery. Clin J Am Soc Nephrol. https://doi.org/10.2215/CJN.00240605.accessed 11 Sep 2016.

15. Bonventre JV, et al. Recent advances in the pathophysiology of ischemic acute renal failure. J Am Soc Nephrol. 2003 Aug;14(8):2199-210. doi:10.1097/01.asn.0000079785.13922.f6.

16. Brown JR, Kramer RS, Coca SG, Parikh CR. Duration of acute kidney injury impacts long-term survival after cardiac surgery. Ann Thorac Surg. 2010;90:1142-8. doi:10.1016/j.athoracsur.2010.04.039.

17. Hobson CE, Yavas S, Segal MS, Schold JD, Tribble CG, Layon AJ, Bihorac A. Acute kidney injury is associated with increased long-term mortality after cardiothoracic surgery. Circulation. 2009;119:2444-53. doi:10.1161/CIRCULATIONAHA.108.800011.

18. Kama A, Wlodzimirow A, Abu-Hanna M, Slabbekoorn RAFM, Chamuleau, Marcus J, Schultz, Catherine SC. Bouman:A comparison of RIFLE with and without urine output criteria for acute kidney 
injury in critically ill patients. Crit Care 2012 Oct 18;16(5):R200. doi: 10.1186/cc11808.

19. Chew STH, Mar WMT, Ti LK. Association of ethnicity and acute kidney injury after cardiac surgery in a South East Asian population. Br J Anaesth. 2013;110:397-401. doi:10.1093/bja/aes415.

Figures

\section{ROC curve: Creatinine Reduction Ratio for AKI}

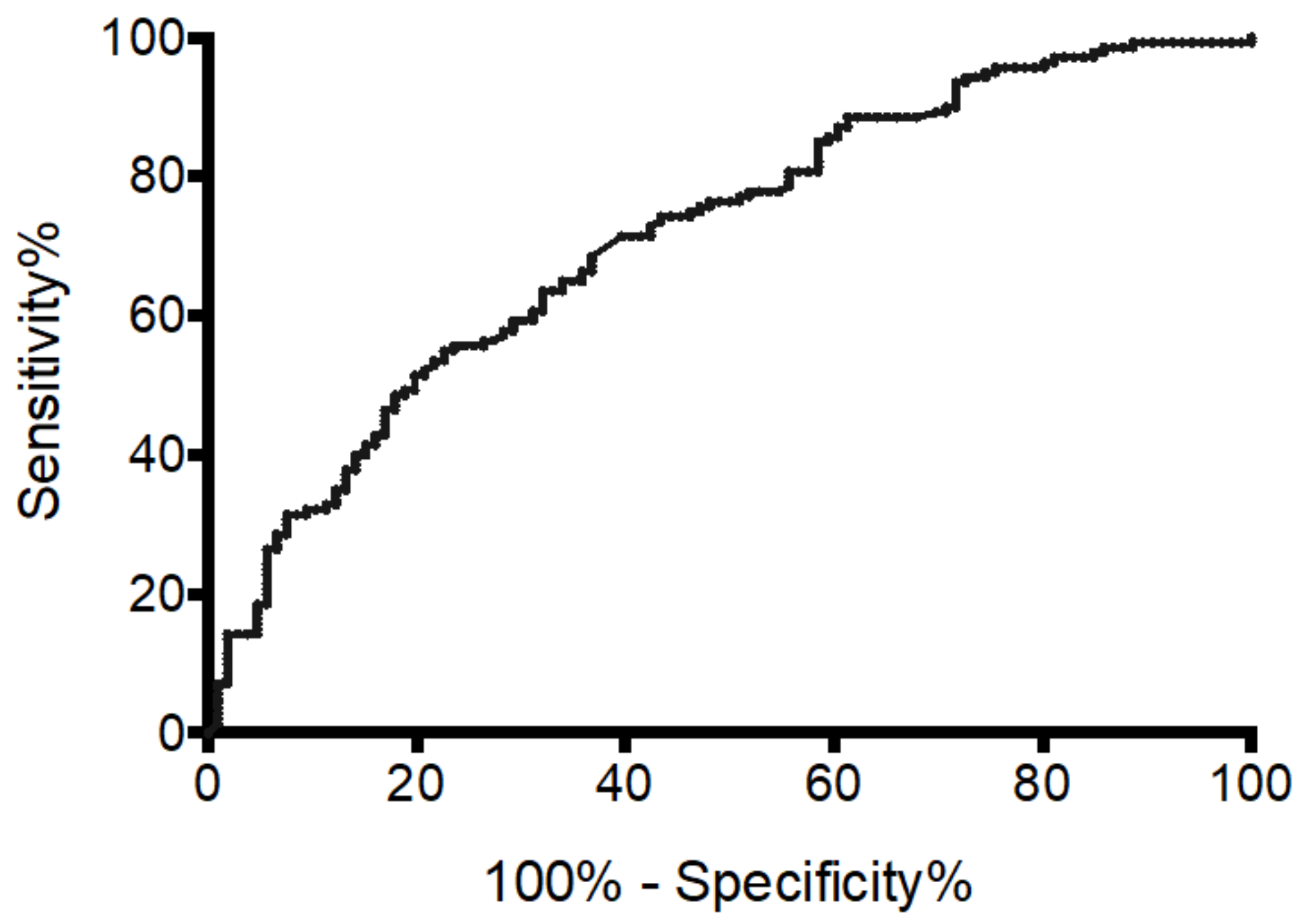

Figure 1

Area under the ROC curve of Creatinine Reduction Ratio for AKI prediction Area under the receiver operating characteristic curve for AKI prediction was 0.714 ( $p<0.001)$. CRR $<20 \%$ : Sensitivity $93.6 \%$, Specificity $28.3 \%$, Likelihood ratio $1.31 \mathrm{CRR}<15 \%$ : Sensitivity $88.6 \%$, Specificity $34.0 \%$, Likelihood ratio 1.34 ROC; receiver operating characteristic, CRR; Creatinine reduction ratio, AKl; acute kidney injury 